\title{
Singen öffnet Herzen und Lungen
}

\section{Wer singen will, braucht ausreichend Luft. Ein britisches Forscherteam hat jetzt analysiert, wie sich ein Gesangs- training auf das Wohlbefinden und die Gesundheit von Menschen mit respira- torischen Krankheiten auswirkt.}

- Die Wissenschaftler um Adam Lewis vom Londoner Imperial College hatten sechs Studien ausgewertet, in denen $\mathrm{Pa}$ rameter wie Gesundheitsstatus, Lungenfunktion und Lebensqualität von Gesangsschülern mit Daten von Personen unter Standardversorgung verglichen wurden. Die Sänger nahmen an einer Initiative namens "Singing for Lung Health “ (SLH) teil, die u.a. von der Britisch Lung Foundation unterstützt wird. Ziel ist es, Menschen mit chronischen Atemwegserkrankungen zu mehr Luft zu verhelfen.

Der regelmäßige Unterricht, in den Übungen zu Körperhaltung und Atemkontrolle einfließen, wird von einem Gesangslehrer geleitet.

Der Analyse zufolge scheint das Singen bei Menschen mit respiratorischen Erkrankungen die Lebensqualität zu verbessern. Positive Auswirkungen zeigten sich auf die körperliche Gesundheit und das Angstniveau. Außerdem wurden

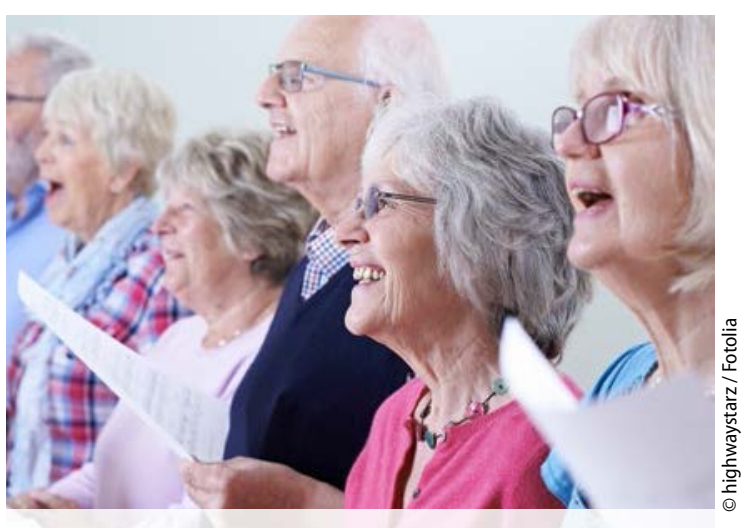

Freude ins Herz, Luft in die Lungen. günstige Auswirkungen auf einzelne Lungenfunktionsparameter beschrieben. Insbesondere COPD-Patienten berichteten darüber, dass ihnen der Unterricht helfe, mit ihrer Erkrankung besser zurechtzukommen.
Die Wirkung der Singtherapie sollte nun in Langzeitstudien weiter untersucht werden.

- Lewis A et al. npj Primary Care Respiratory Medicine 2016; online 1. Dezember 2016, DOI:10.1038/npjpcrm.2016.80

\section{Wurst und Schinken nähren Asthmasymptome}

\section{Gepökelte Fleischprodukte sollten nicht allzu oft auf dem Speiseplan von Asthmapatienten stehen: Einer neuen Studie zufolge bewirken sie eine Zu- nahme von Asthmabeschwerden.}

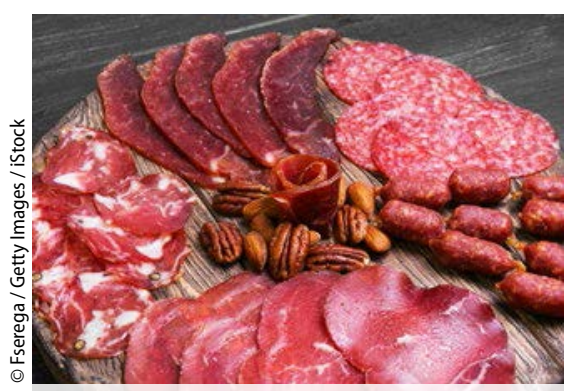

Je mehr Wurst, desto mehr Atemnot.
_ Regelmäßig gepökelte Fleischerzeugnisse zu essen, gilt in vielerlei Hinsicht als gesundheitsschädlich: Die WHO hat die Produkte als karzinogen eingestuft. Auch das Risiko für KHK und Diabetes ist erhöht. Neu auf der Liste sind Asthmabeschwerden. Laut einer prospektiven Studie aus Frankreich sind Asthmapatienten, die reichlich Wurst und Schinken essen, häufiger von einer Verschlechterung betroffen.

Die zugrunde liegenden Daten stammen aus einer Kohortenstudie, an der Asthmapatienten, erstgradige Verwandte und Kontrollpersonen beteiligt waren. Die 971 Teilnehmer beantworteten ausführliche Ernährungsfragebogen. Während der Nachbeobachtungszeit von im
Mittel sieben Jahren kam es bei 20\% der Teilnehmer zu einer Zunahme von Asthmabeschwerden wie Engegefühl in der Brust oder Atemnot.

Der Anteil der Personen mit einer Verschlechterung der Symptome lag je nach Konsum von Wurst und Schinken ( $<1,1-3,9$ oder $\geq 4$ Portionen pro Woche) bei $14 \%, 20 \%$ oder $22 \%$. Auch nach Adjustierung für Risikofaktoren wie Rauchen sowie für Alter und körperliche Aktivität ergab sich ein direkter Effekt: Das Risiko für eine Verschlechterung des Asthmas lag in der Gruppe mit dem höchsten Verzehr um 76\% höher als in der Gruppe mit dem geringsten. - bs - LiZ et al. Thorax, online 20. Dezember 2016 